\title{
A CTLA-4 blocking strategy based on Nanobody in dendritic cell-stimulated cytokine-induced killer cells enhances their anti-tumor effects
}

Wu Wang ${ }^{1,2,3+}$, Xi Wang ${ }^{4 \dagger}$, Wenli Yang ${ }^{5+}$, Kai Zhong ${ }^{6 \dagger}, \mathrm{Na} \mathrm{He}^{2+}$, Xuexia $\mathrm{Li}^{2+}$, Yanyang Pang ${ }^{3+}, \mathrm{Zi} \mathrm{Lu}^{7 \dagger}$, Aiqun Liu ${ }^{1,8^{*}}$ and Xiaoling Lü ${ }^{1^{*}}$

\begin{abstract}
Background: Cytokine-induced killer cells induced with tumor antigen-pulsed dendritic cells (DC-CIK) immunotherapy is a promising strategy for the treatment of malignant tumors. However, it sefficacy is restricted by the immunosuppression, which is mediated by the cytotoxic T-lymphocyte-associated antigen-4 (CTLA-4) pathway. In order to overcome the negative co-stimulation from these T cells, we screened a nanobody targeted for CTLA-4 (Nb36) and blocked the CTLA-4 signaling with Nb36.

Methods: Peripheral blood mononuclear cells (PBMCs) were collected from healthy donors to beused to induce CIK cells in vitro, after which they were co-cultured with DC cells that had received tumor antigens. In addition, we tested whether blocking CTLA-4 signaling with Nb36 could promote in vitro DC-CIK cells proliferation, proinflammatory cytokine production and cytotoxicity, or not. For the in vivo experiments, we constructed a subcutaneously transplanted tumor model and placed it in NOD/SCID mice to verify the anti-tumor effect of this therapy.

Results: After stimulation with Nb36, the DC-CIK cells presented enhanced proliferation and production of IFN- $\gamma$ in vitro, which strengthened the killing effect on the tumor cells. For the in vivo experiments, it was found that Nb36-treated DC-CIK cells significantly inhibited the growth of subcutaneously transplanted livercancer tumors, as well as reduced the tumor weight and prolonged the survival of tumor-bearing NOD/SCID mice.

Conclusions: Our findings demonstrated that in response to CTLA-4 specific nanobody stimulation, DC-CIK cells exhibited a better anti-tumor effect. In fact, this Nb-based CTLA-4 blocking strategy achieved an anti-tumor efficacy close to that of monoclonal antibodies. Our findings suggest that DC-CIK cells + Nb36 have the potential to treat malignant tumors through in vivo adoptive therapy.
\end{abstract}

Keywords: Nanobody, Cytotoxic T-lymphocyte antigen-4, Cytokine-induced killer cells

\footnotetext{
* Correspondence: liuaiqun_2004@163.com; luwuliu@163.com

'Wu Wang, Kai Zhong, XiWang, Wenli Yang, Na He, Xuexia Li, Yanyang Pang and Zi Lu contributed equally to this work.

${ }^{1}$ International Nanobody Research Center of Guangxi, College of Stomatology, Guangxi Medical University, Nanning, Guangxi 530021, China Full list of author information is available at the end of the article
}

C C The Author(s). 2021 Open Access This article is licensed under a Creative Commons Attribution 4.0 International License, which permits use, sharing, adaptation, distribution and reproduction in any medium or format, as long as you give appropriate credit to the original author(s) and the source, provide a link to the Creative Commons licence, and indicate if changes were made. The images or other third party material in this article are included in the article's Creative Commons licence, unless indicated otherwise in a credit line to the material. If material is not included in the article's Creative Commons licence and your intended use is not permitted by statutory regulation or exceeds the permitted use, you will need to obtain permission directly from the copyright holder. To view a copy of this licence, visit http://creativecommons.org/licenses/by/4.0/. The Creative Commons Public Domain Dedication waiver (http://creativecommons.org/publicdomain/zero/1.0/) applies to the data made available in this article, unless otherwise stated in a credit line to the data. 


\section{Background}

Cancer immunotherapy obtains beneficial effects by mediating tumor cell regression, which relies on the activation, persistence and targeting of anti-tumor $\mathrm{T}$ cells [1]. Cytokine-induced killer cells (CIK) are immunologically active cells that have been expanded in vitro, and which have both the strong cytotoxicity of T-lymphocytes and the non-MHC-restricted killing characteristics of NK cells [2]. As the most powerful antigen-presenting cells, dendritic cells (DCs) can capture the antigen and then present it to the surface of a responder (such as CIK), thereby activating the antigen-specific immune response and improving the function of the effector cells. DC-CIK therapy has the potential for both MHC-unrestricted and tumorspecific activity [3]. However, during solid tumor therapy, it was found that an immunosuppression of the tumor's microenvironmentled to the restriction of the cytotoxicity and proliferation of DC-CIKs in vivo [4]. In addition to dysfunctional stromal cells in the tumor microenvironment, there are also various regulatory T-cells, myeloid-derived suppressor cells, and up-regulated tumor suppressor molecules such as cytotoxic T-lymphocyte-associated antigen-4 (CTLA4 ) and programmed cell death protein-1 (PD-1) $[5,6]$. This complex tumor microenvironment is a hotbed for tumor development and leads to the exhaustion of infused DC-CIK cells, and is therefore the main mechanism limiting the efficacy of DC-CIK adoptive therapy [7].

CTLA-4 is a protein receptor mainly expressed in both activated and regulatory T-cells. CTLA-4 competes with CD28 for ligand binding and emits inhibitory signals to attenuate T-cell activation [8]. Expressions of CTLA-4 areusually up-regulated with the continuous activation of T-cells. CTLA-4 can be combined with molecules of the B7 family on the DCs' surface as a co-stimulatory signal to inhibit the proliferation, activation and cell cycle of the T-cells. This leads to decreased secretion of cytokines such as IL-2, IL-4, IFN- $\gamma$ and decreased expression of IL-2 receptors. This opens a window for tumors to escape fromimmune surveillance by negatively regulating $\mathrm{T}$ cellproliferation [8-10]. As one of the most important immunosuppressive receptors, CTLA-4-targeted immune checkpoint inhibitors are also an important and much researched topic in tumor immunotherapy. The blockade of the CTLA-4 signaling with monoclonal antibodies leads to an enhancement of the antitumor immune response, meaning that it has become a potential tumor immunotherapy strategy and is currently undergoing a number of clinical investigations [11, 12]. However, this strategy presents many limitations, including the non-specific binding of monoclonal antibodies to normal tissue and heterogeneous tumor antigens, and poor penetration of antibodies in the tumor microenvironment. Therefore, finding a way to develop novel antibodies with high efficiency and low toxicity is a matter of great urgency [13].

A nanobody $(\mathrm{Nb})$ is a special single domain antibody which is derived from camelids. These animals naturally possess a special single-chain antibody (lacking light chain and $\mathrm{CH} 1$ ), and $\mathrm{Nb}$ is obtained after cloning its variable regions; which is in fact the smallest antigen-binding unit ever found [14]. Its simple molecular structure allows $\mathrm{Nb}$ to bind to epitopes that are not easily accessible to traditional antibodies [15]. In addition, $\mathrm{Nb}$ has a strong tolerance to changes in temperature and $\mathrm{pH}$, and furthermore, its stable conformation allows it to be taken orally. Compared with conventional antibodies, $\mathrm{Nb}$ has high specificity, good physical and chemical stability, high yield, low cost, and lacks immunogenicity, which make it very suitable to a clinical setting [16]. In previous studies, we used phage display technology to obtain a set of CTLA-4 specific Nb. This anti-CTLA$4 \mathrm{Nb}$ (Nb36) can bind to CTLA-4 epitopes on the surface of activated T-cells in vitro $[17,18]$. To further investigate its antitumor activity in this study, we have used these novel antibodies to block CTLA-4 signaling in activated DC-CIK cells, thereby promoting the ability of DC-CIK to survive, proliferate and infiltrate (Fig. 1).

\section{Materials and methods}

\section{Animals and cell culture}

Female NOD/SCID mice, aged 4-6 weeks, were purchased from Beijing Vital River Lab of Animal Technology (Beijing, China) and raised in an SPF environment. All protocols were approved by the Animal Ethics Committee of Hainan Medical University. HepG2 (hepatoma carcinoma) and A549 cells (lung carcinoma) were purchased from the International Nanobody Research Center of Guangxi and cultured in DMEM supplied with $10 \%$ fetal bovine serum (FBS) and penicillin/streptomycin in $37^{\circ} \mathrm{C}, 5 \% \mathrm{CO}_{2}$.

\section{Antibodies and Nanobodies}

The anti-CTLA-4 Nb (Nb36) was developed in our previous study [17]. Anti-CTLA-4 mAb (Rabbit monoclonal, UC10-4F10-11), anti-CD3 mAb (Rabbit monoclonal, SP7), anti-CD56 mAb (Rabbit monoclonal, EPR21827), anti-CD80 mAb (Rabbit monoclonal, EPR1157(2)), anti-CD83 mAb (Rabbit monoclonal, EPR23809-19) and anti-MHC II mAb (Rabbit monoclonal, 6C6) were all purchased from Abcam (Cambridge, UK). 


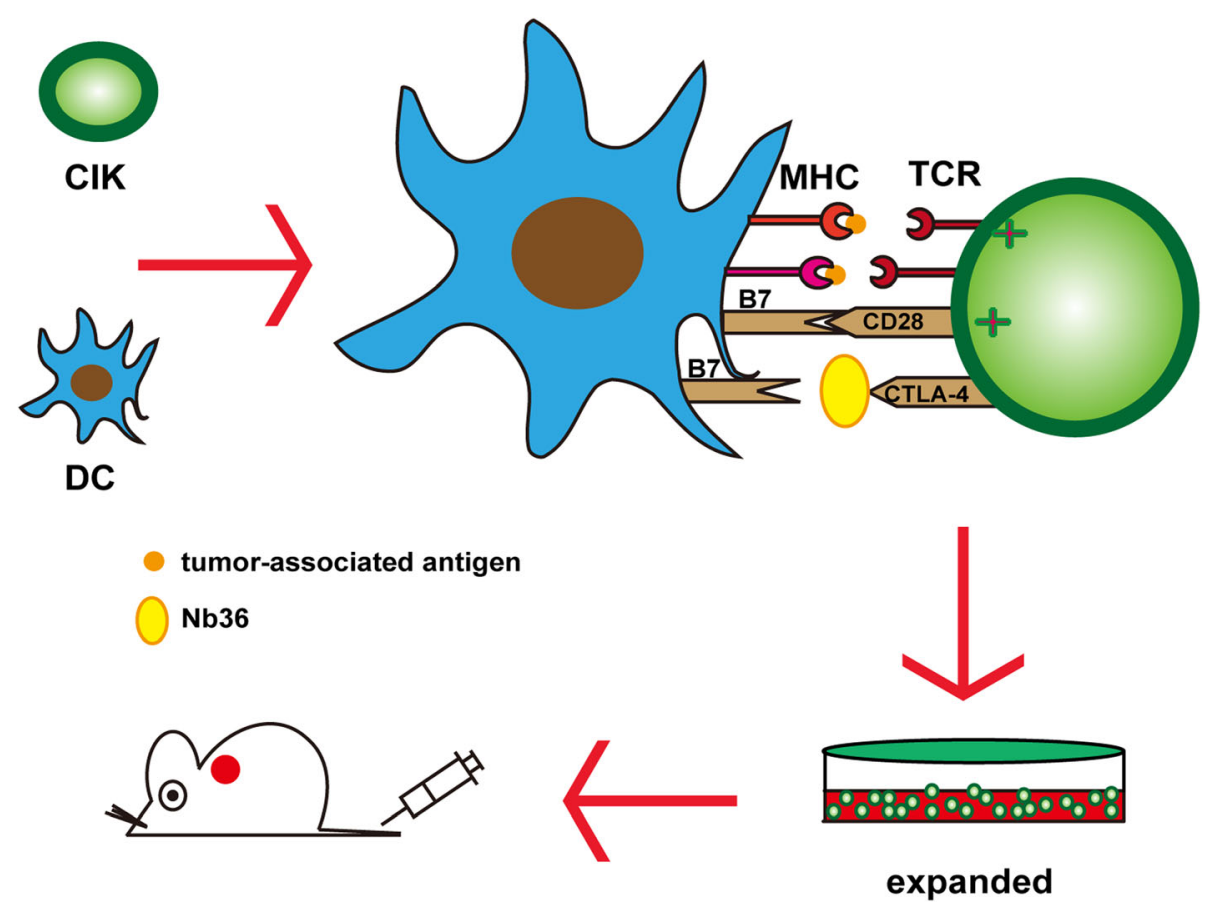

Fig. 1 Therapy strategy with DC-CIK cells+anti-CTLA-4 nanobodies. DC-CIK cells were first generated. Then, the nanobodies against CTLA-4 (Nb36) eliminated immunosuppression via blocking CTLA-4-mediated negative co-stimulation in the effector DC-CIK cells. Finally, DC-CIK cells were transferred to kill the tumor cells

\section{Generation of CIK and DCs}

Density gradient centrifugation was used to collect peripheral blood mononuclear cells (PBMCs) from healthy donors, after which they were cultured in Roswell Park Memorial Institute (RPMI)-1640 medium (Gibco), containing 10\% FBS and penicillin/streptomycin. Following $4 \mathrm{~h}$ of culture, the suspended cells (T-cells) were grown to generate CIK cells in RPMI-1640 with 10\% FBS, containing $500 \mathrm{ng} / \mathrm{ml}$ anti-CD3 antibody, $100 \mathrm{U} / \mathrm{ml}$ IFN- $\gamma$ (Servicebio, Wuhan, China) and $10 \mu \mathrm{g} / \mathrm{ml}$ polyhydroxyalkanoates (Solarbio, Beijing, China). The cell concentration was adjusted to $1 \times 10^{6}$ cells $/ \mathrm{ml}$. In addition, the adhered cells $(1 \times$ $10^{5}$ cells $/ \mathrm{ml}$ ) were used for dendritic cell differentiation via culturing in RPMI-1640 (containing 10\% FBS and penicillin/streptomycin), supplemented with $1000 \mathrm{U} / \mathrm{ml}$ recombinant human GM-CSF (rhGM-CSF; R\&D, MN, US) and $500 \mathrm{U} / \mathrm{ml}$ rhIL-4 (R\&D, MN, US) for 7 days. Next, A549 and HepG2 cells were lysed (repeated freeze-thaw procedure), and the supernatant was obtained as the tumor antigen. On day 8, the supernatant as well as10 ng/ml TNF$\alpha$ and $10 \mathrm{ng} / \mathrm{ml}$ IL- $1 \beta$ were added to the DCs' medium, after which the culture was maintained for 2 days. On day 10, DCs and CIKs were co-cultured at a ratio of 1:10 for 2 days (RPMI-1640 with 10\% FBS containing $500 \mathrm{ng} / \mathrm{ml}$ anti-CD3 antibodies). On day 12, we added the Nb36 to the DC-CIK cells to mediate the CTLA-4 blockade [19]. This study has been approved by the local ethics committee of Hainan Medical University.

\section{Phenotypic analysis of CIK and DCs}

On day 7, flow cytometry was used to analyze the HLADR, CD80 and CD83 expression of DCs. Following this, a phenotypic analysis of DC-CIK cells, including CD56 and CD3 was performed by flow cytometry on day 14 (Backman CytoFlex S).

\section{Cell proliferation assay}

In vitro CFSE staining was used to determine the proliferation of DC-CIK cells following their stimulation by tumor antigens. Individual groups of DC-CIK cells were stimulated with $\mathrm{Nb36}(50 \mu \mathrm{g} / \mathrm{ml})$, anti-CTLA-4 mAb $(50 \mu \mathrm{g} / \mathrm{ml})$, and anti-CD105 $\mathrm{Nb}$ (Isotype Control, $50 \mu \mathrm{g} /$ $\mathrm{ml})\left(1 \times 10^{6}\right.$ cells/tube $)$, after which they were labeled with CFSE (Sigma-Aldrich, MO, US) at $37^{\circ} \mathrm{C}$ for $5 \mathrm{~min}$. After washing with PBS, the cells were co-cultured with the same number of HepG2 cells that were exposed with a cell irradiator to $100 \mathrm{~Gy}$ of radiation for $120 \mathrm{~h}$. The suspended CIK cells were harvested, and the percentage of proliferative CIK cells was determined by flow cytometry (Backman CytoFlex S).

\section{Cytotoxicity assay}

We next assessed the cytotoxicity of DC-CIK cells against HepG2 and A549 cells in vitro. After being stimulated with Nb36, anti-CTLA-4 mAb, or anti-CD105 $\mathrm{Nb}$, the DC-CIK cells were collected and mixed with HepG2, or with A549 cells with E:T ratios of 5:1, 10:1 or 
20:1. The target cells with PKH26 staining were cocultured for $6 \mathrm{~h}$ at $37^{\circ} \mathrm{C}, 5 \% \mathrm{CO}_{2}$. The PKH26-labeled cells were stained with propidium iodide (PI) and flow cytometry was used to determine the percentage of $\mathrm{PKH} 26^{+} \mathrm{PI}^{+}$dead cells (Backman CytoFlex S).

\section{ELISA and ELISPOT assays}

DC-CIK cells and T-cells (control, $1 \times 10^{5} /$ well) were co-cultured with HepG2 cells $\left(1 \times 10^{5} /\right.$ well $)$ for $16 \mathrm{~h}$. We used ELISA to detect the supernatant's levels of IL2 , TNF- $\alpha$ and IL-10. Meanwhile, ELISPOT assay was performed to detect the ratio of effector cells which specifically secreted IFN- $\gamma$. Briefly, DC-CIK cells and T-cells (control, $3 \times 10^{5} /$ well) were co-cultured in triplicate with the HepG2 cells $\left(1 \times 10^{5} /\right.$ well $)$ that had been irradiated in plates precoated with anti-IFN-yat $37^{\circ} \mathrm{C}$ for $12 \mathrm{~h}$. After being washed with PBS, biotinylated anti-IFN- $\gamma$ were added and reacted at $4{ }^{\circ} \mathrm{C}$ for $12 \mathrm{~h}$. Then, the IFN- $\gamma$ specific immunocomplex was analyzed by streptavidin ap and observed in the substrate solution (BCIP/NBT). The number of spots was detected using a CTL ImmunoSpot S6 Ultimate-V analyzer.

\section{Xenograft experiments in mice}

For xenograft experiments, each NOD/SCID mouse was implanted with HepG2 cells $\left(2 \times 10^{6} /\right.$ injection $)$ via subcutaneous injection in the left armpit. Once the tumors reached a volume of $100 \mathrm{~mm}^{3}$, these tumor-bearing mice were injected three times every 7 days through the tail vein with $5 \times 10^{6} \mathrm{DC}-\mathrm{CIK}$ cells, Nb36-treated DC-CIK cells, anti-CTLA-4 mAb-treated DC-CIK cells, antiCD105 Nb-treated DC-CIK cells, or PBS. The tumor volume was then recorded with a vernier caliper every 3 days to evaluate tumor growth. Tumor volume (V) was calculated according to the formula: $\mathrm{V}=0.5 \mathrm{ab}^{2}$, where a is the largest diameter, and $b$ is the perpendicular diameter.

\section{Immunohistochemistry}

The tumor tissue samples were fixed with $10 \%$ neutral formalin before paraffin embedding, and the slice thickness was $4-\mu \mathrm{m}$. Next, these sections were incubated with anti-ki67 monoclonal antibodies overnight at $4{ }^{\circ} \mathrm{C}$. After washing twice, the sections were further incubated with the HRP-labeled secondary antibodies. Images were obtained with a microscope (Nikon, Japan).

\section{Statistical analysis}

The FACS data was analyzed using FlowJo v10.07 software, while GraphPad Prism software 6.0 was utilized for the statistical analysis of data. The differences found between the experiments' different groups of data were analyzed using One-way ANOVA alongside Tukey's multiple comparison. The tumor growth curve was evaluated using two-way ANOVA with corrections for Tukey's multiple comparison. The survival curve for themice in the different groups was analyzed using KaplanMeier analysis (log-rank test).

\section{Results}

\section{Characterization of the Nb36-treated DC-CIK cells}

Flow cytometry was used to monitor the immunophenotype of the ex vivo cultivated cells. The high expression of HLA-DR (89.4 \pm 3.55\%), co-stimulatory molecules CD80 (99.2 $\pm 0.7 \%)$, and the maturation marker CD83 $(47.3 \pm 2.38 \%)$ found on day 7 demonstrated the maturation of the DCs (Fig. 2A). These DCs were stimulated by tumor antigens and incubated with CIK. The CIK cells had the $\mathrm{CD}^{+} \mathrm{CD}^{+} 6^{+}$phenotype in the $\mathrm{DC}-\mathrm{CIK}+\mathrm{Nb} 36$ group with a total cells median percentage of $46.43 \%$ (range 43.1-48.4\%), which was significantly higher than the untreated DC-CIK group (33.46\%, range 32.1-36.1\%) and close to the CTLA-4 mAb treated group (46.16\%, range $42.8-49.42 \%$ ) (Fig. $2 \mathrm{~B}$ ). These results indicate that DC-CIK cells have a more efficient activation and expansion when CTLA-4 signaling is blocked.

\section{Blocking CTLA-4 signaling with Nb36 promoted proliferation of DC-CIK cells in vitro}

We tested whether blocking CTLA-4 signaling with antiCTLA-4 Nb could promote DC-CIK cells proliferation and pro-inflammatory cytokine production in vitro. The results showed that $120 \mathrm{~h}$ of stimulation with tumor cells (HepG2) significantly promoted the proliferation of CFSElabeled DC-CIK cells in the DC-CIK + Nb36 group, promoting it far more than in the DC-CIK $(p<0.001)$ and DC-CIK + CTLA-4 mAb $(p<0.05)$ groups (Fig. 3A-B). In addition, longitudinal measurements of Nb36-treated DCCIK cells proliferation suggested that the number of CFSE-labeled DC-CIK cells was significantly greater than in other groups, except for in the DC-CIK + CTLA-4 mAb group. After 11 days of cultivation, the number of DC-CIK cells in the DC-CIK + Nb36 group had increased by 180 times (Fig. 3C). These data therefore indicate that blocking CTLA-4 signaling with Nb36 further activates DCCIK cells and promotes cell proliferation in vitro.

\section{Blocking CTLA-4 signaling with Nb36 facilitated the tumor cell killing of DC-CIK cells in vitro}

A cytotoxicity assay was performed to examine the impact Nb36-treated DC-CIK cells had on target cell killing. The results indicated that the effector-target ratios of (E:T) 5:1, 10:1 or 20:1 had a much higher killing effect on HepG2 and A549 cells for the effector cells in the DC-CIK+ $\mathrm{Nb36}$ group than for theT-cell, DC-CIK and DC-CIK + CD105 Nb cells, with these having low or little cytotoxicity against such target cells (Fig. 3D-E). Hence, cells in the DC-CIK $+\mathrm{Nb36}$ group were shown to effectively and 


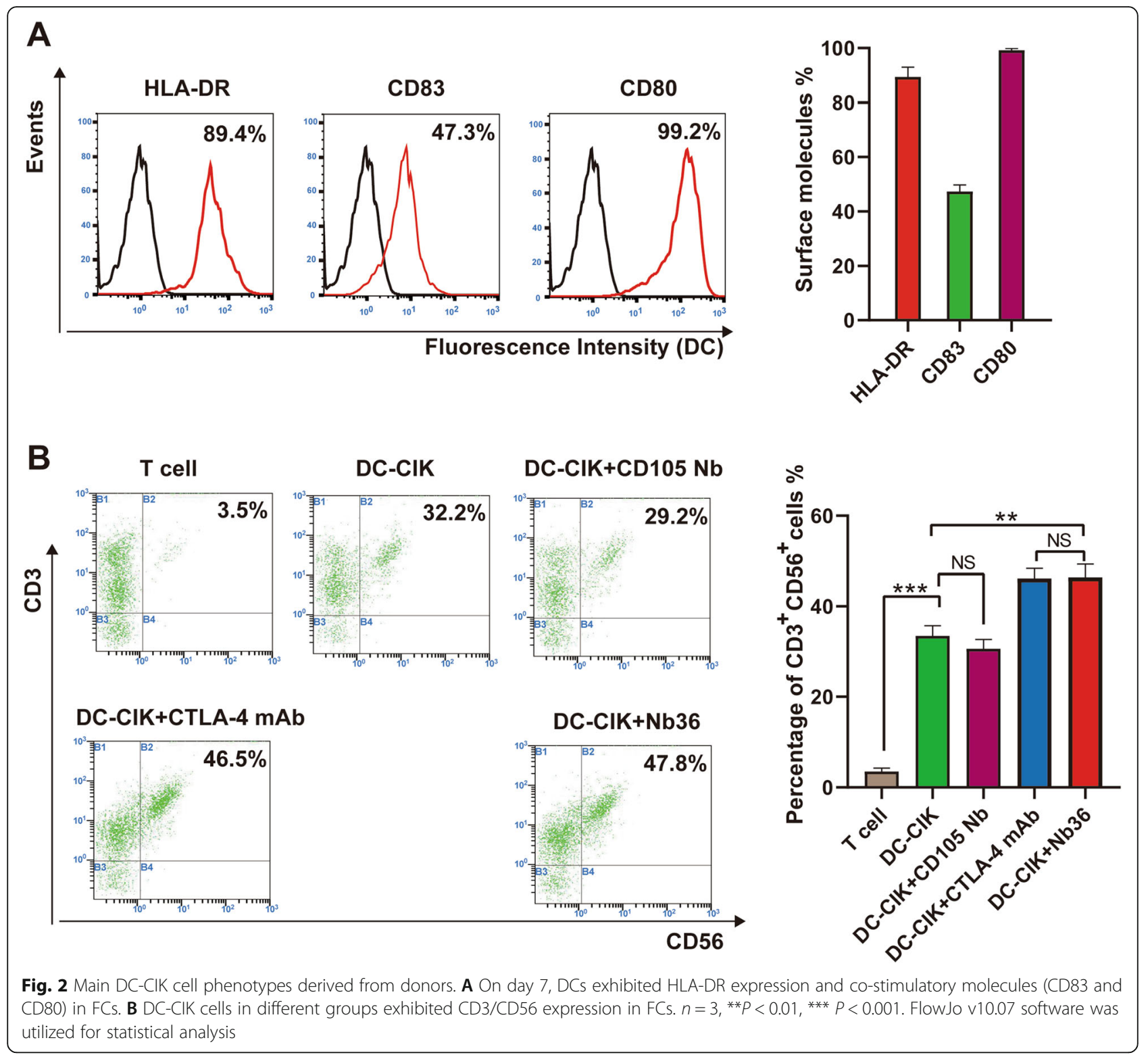

specifically kill target cells in vitro, with their efficiency being close to the DC-CIK + CTLA-4 mAb group.

\section{Blocking CTLA-4 signaling with Nb36 stimulated DC-CIK} cells activation and pro-inflammatory cytokine production in vitro

We tested whether blocking CTLA-4 signaling with Nb36 could stimulate DC-CIK cells activation and proinflammatory cytokine production in vitro. To test this, groups of DC-CIK cells were stimulated with the same number of HepG2 cells for $12 \mathrm{~h}$. Then, the supernatants' levels of IL-2, TNF- $\alpha$ and IL-10 were measured by ELISA. We found that after being challenged with target cells, the levels of IL-2 and TNF- $\alpha$ in the supernatants belonging to the cultured Nb36-treated DC-CIK group were significantly higher than those in the DC-CIK and anti-CD105 Nb-treated DC-CIK groups (Fig. 4A). However, no significant difference could be seen in the expression of IL-10 among the different groups (Fig. 4A). ELISPOT revealed that after being challenged with HepG2 cells, the number of IFN- $\gamma$-secreting spot forming cells in the Nb36-treated DC-CIK group was significantly greater than those found in the T-cells, DC-CIK cells and DC-CIK + CD105 Nb groups (Fig. 4B). These data therefore demonstrate that Nb36-treated DC-CIK cells have activated and secrete higher levels of proinflammatory cytokines after being challenged with target cells. 


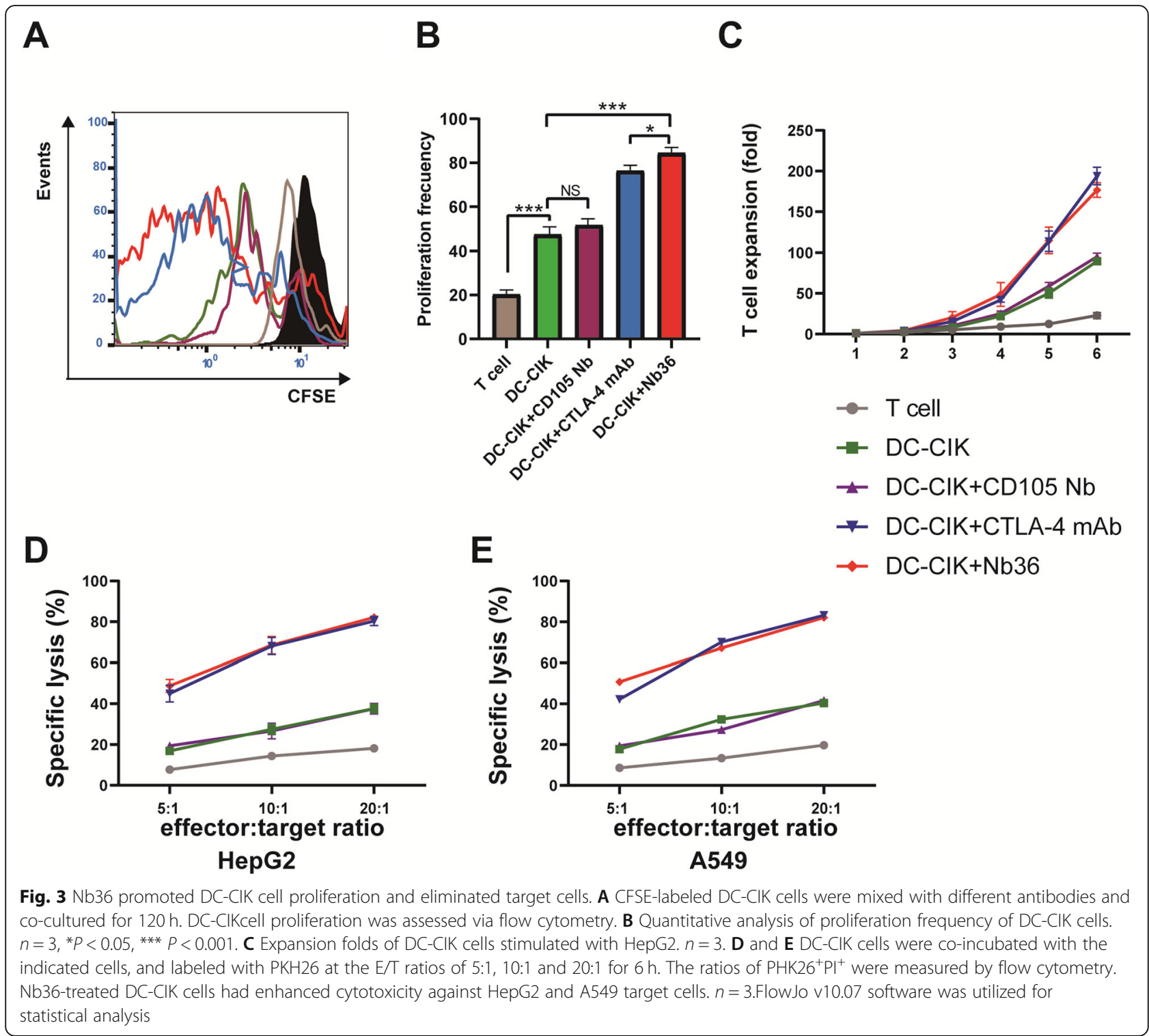

\section{$\mathrm{Nb36}$ increased the anti-tumor efficacy of DC-CIK cells in vivo}

We examined the effects of Nb36-treated DC-CIK cells in vivo. It was found that treatment with DC-CIK Cells+ $\mathrm{Nb36}$ was able to significantly reduce the volume and weight of the tumors in mice, and that it prolonged the survival of tumor-bearing mice when compared with PBS, T-cells, DC-CIK and DC-CIK + CD105 Nb groups (Fig. 5A-B). Similarly, the immunohistochemical analysis of the xenograft tumor tissue revealed that the number of anti-Ki67 cells in the mice receiving Nb36-treated DC-CIK cells was significantly lower than in any of the other groups (Fig. 5C). These data therefore indicate that treatment with Nb36-treated DC-CIK cells inhibits the growth of implanted tumors in mice.

\section{Discussion}

Compared with traditional methods, tumor immunotherapy has become a promising strategy for the treatment of malignant tumors. Numerous clinical studies have shown that an activation of the anti-tumor effector cells for adoptive introduction into the patient could improve the prognosis $[20,21]$. Thisis very important for the $\mathrm{CD}^{+} \mathrm{CD}^{+}$cytotoxic $\mathrm{T}$-cells subgroup, which isinduced by DCs in specific cellular immune responses. After induction, DC-CIK cells have the potential for both MHC-unrestricted and tumor-specific cytotoxicity [22]. Induction with anti-CTLA-4 monoclonal antibodies was shown to promote DC-CIK cell proliferation and differentiation into $\mathrm{CD}^{+}{ }^{+} \mathrm{CD} 56^{+} \mathrm{NK}$ T-cells, which is the main effector of DC-CIK cells, providing synergistic 


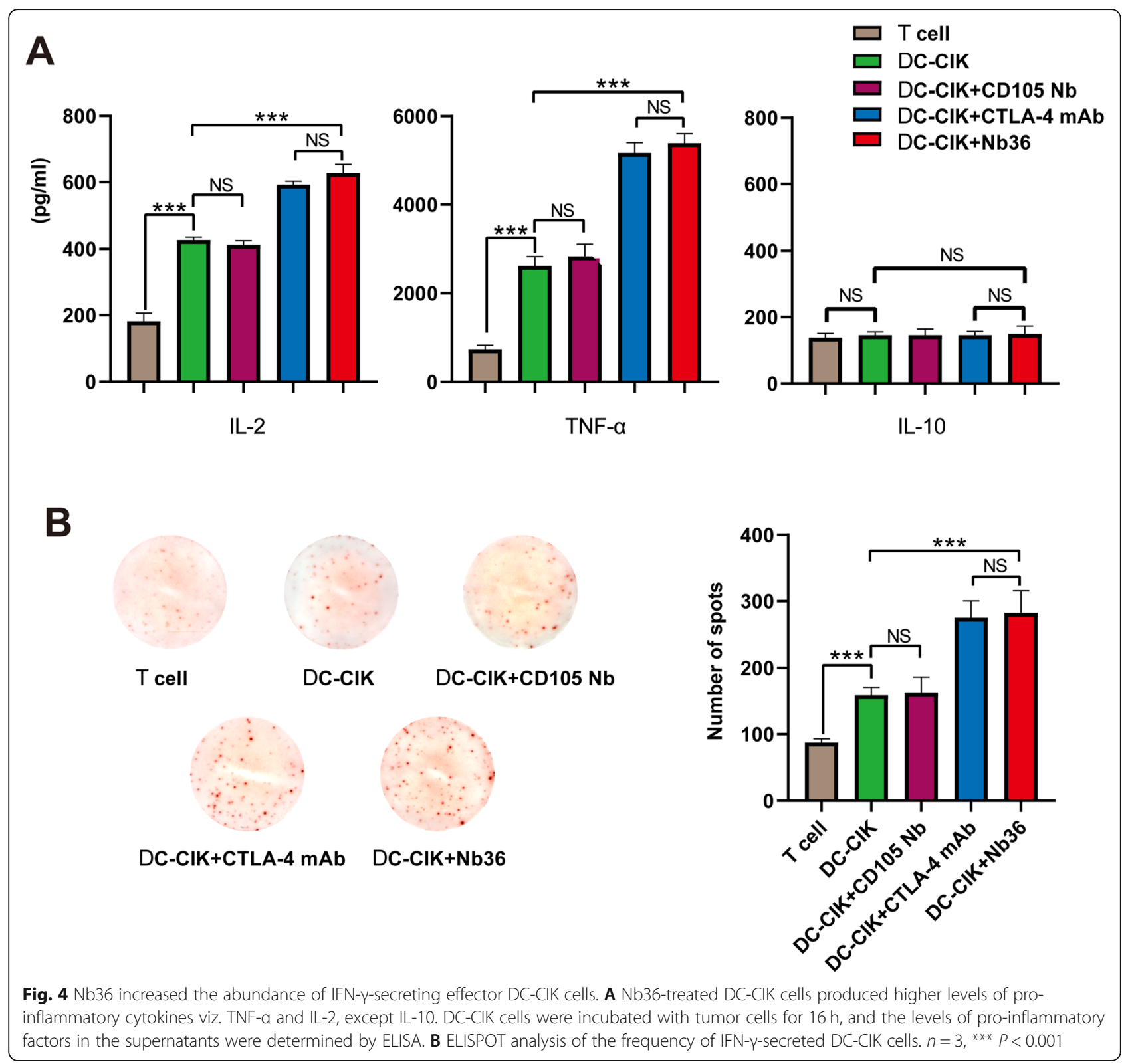

antitumor effects by up-regulating the secretion of proinflammatory cytokine and decreasing the production of immunosuppressive cytokines in vivo [23].

The CTLA-4 pathway delivers inhibitory signals that negatively regulate effector T-cells to inhibit their activation. Several studies have revealed that the effects of DC-CIK cells are combined with the effects from the monoclonal antibodies targeting CTLA-4 [24]. However, the large molecular weight of monoclonal antibody drugs limits their penetration and concentration in solid tumors, which results in the tumor treatment's poor effectiveness. Moreover, the high costs involved prevents its wide spread usage [25]. In order to overcome the above disadvantages, novel antibodies including single chain antibodies, genetically-engineered antibodies and nanobodies have been developed [26]. Specifically, and in view of the unique structural and molecular characteristics of $\mathrm{Nb}, \mathrm{Nb}$-targeting CTLA-4 may be an effective strategy to enhance the effect of DC-CIK cells.

Our study successfully obtained CIK cells from PBMCs, as was shown after the 12-day in vitro expansion where anti-CD3 antibodies, IFN- $\gamma$ and PHA were sequentially added. Previous studies have shown that CIK cells have the dual properties of NK and Tcells [27], which is consistent with our research, as shown by the positive expression of CD3 and CD56 immune markers. DCs are the most significant antigenpresenting cells that stimulate effector $\mathrm{T}$-cells to enhance the immune response. We lysed all the tumor cells in order to generate tumor antigens to then induce 


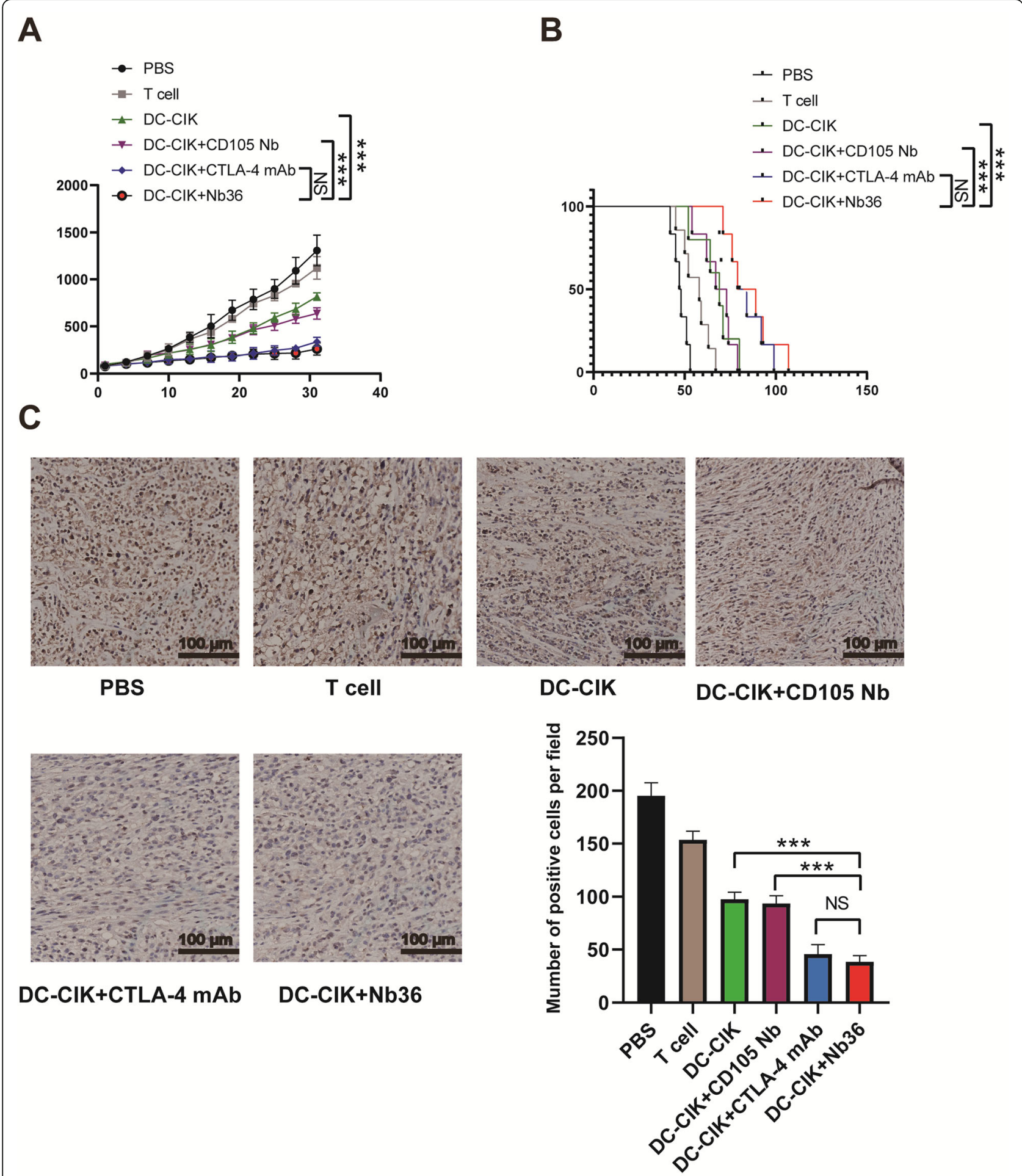

Fig. 5 In vivo antitumor activities of Nb36-treated DC-CIK cells in the established subcutaneous human tumor xenografts. A The tumor growth curves. Nb36-treated DC-CIK cells significantly reduced tumor volume. $n=6$, *** $P<0.001$. B The survival of tumor-bearing mice. Nb36-treated DCCIK cells prolonged the survival of tumor-bearing mice. $n=6,{ }^{* * *} P<0.001$. C Quantitative analysis of KI-67 expression. Data are representative images (magnification $\times 400$ ) or expressed as the mean \pm SD from 5 randomly selected fields of thin tumor sections. $n=6,{ }^{* * *} P<0.001$ 
DC maturation. Compared with the method of stimulating specific tumor antigens, this method can stimulate immunity against the entire tumor antigen, thereby inducing a more complete cytotoxicity [28]. We found that the proliferation activity of CIK cells was enhanced after DC co-cultivation, which became more pronounced as the CTLA-4 was blocked. Compared with the control group, the levels of pro-inflammatory cytokines viz. TNF- $\alpha$ and IL-2, except IL-10, in the DC-CIK + Nb36 group's culture medium, had all increased following the co-culture with target cells. The in vivo treatment of Nb36-treated DC-CIK cells displayed an inhibited tumor growth and prolonged the survival of human tumor xenograft mice. The immunohistochemical analysis displayed a decreased number of cells with proliferative marker Ki67. Accordingly, our study showed that Nb36treated DC-CIK cells effectively killed target cells both in vitro and in vivo. By binding to CTLA-4 on the surface of the effector lymphocytes, Nb36 was able to increase the activation of DC-CIK cells, with stronger cytotoxicity and anti-tumor effects. This CTLA-4 blocking strategy is expected to be widely used as an alternative for anti-tumor therapy due to the high prices of anti-CTLA-4 mAb drugs. In our next study, we aim to look at improving Nb36's anti-tumor activity by adding FC fragments and carrying nanomaterials, as well as trying to apply Nb36 to anti-tumor therapy in vivo.

\section{Conclusions}

This is the first study to report on the application of nanobodies as CTLA-4 blockers to enhance the efficacy of DC-CIK cells. This preclinical study presents a new strategy and a promising prospect for the immunotherapy of malignant tumors. The results encourage further research on the efficacy of patient-derived DC-CIK cells combined with anti CTLA-4 nanobodies for the treatment of malignant tumors.

\section{Abbreviations}

Nb: Nanobody; DC-CIK: Cytokine-induced killer cells which were induced with tumor antigen-pulsed dendritic cells; CTLA-4: Cytotoxic T lymphocyteassociated antigen-4; PBMC: Peripheral blood mononuclear cells

\section{Acknowledgments}

Not applicable.

\section{Authors' contributions}

Aiqun Liu, Xiaoling Lu and Wu Wang conceived and designed the present study. Wenli Yang, Na He, Xuexia Li and Yanyang Pang performed the molecular and cellular experiments in vitro; Kai Zhong, Xi Wang and Zi Lu performed in the analysis of in vivo mouse model. Wu Wang managed the Written of the manuscript. The authors read and approved the final manuscript.

\section{Funding}

This work was supported, in part, by grants from Scientific Research Project of Higher Education Institutions of Hainan Province (grant no. Hnky2020-40); Research start-up fund of Hainan Medical University (grant no. XRC190028); Natural Science Foundation of Hainan Province (grant no. 819MS060).

\section{Availability of data and materials}

The datasets used and/or analyzed during the current study are available from the corresponding author on reasonable request.

\section{Declarations}

Ethics approval and consent to participate

Ethical approval was obtained for collection of human PBMC samples from the local ethics committee of Hainan Medical University and in accordance with the ethical standards of the institutional and/or national research committee and with the 1964 Helsinki declaration and its later amendments or comparable ethical standards. Written informed consent was signed all volunteer. All procedures involving animal care and use were approved by the Animal Ethics Committee of Hainan Medical University and were in accordance with the National Policy and Regulations on Use of Laboratory Animals. All animal experiments were carried out in compliance with the ARRIVE guidelines.

Consent for publication

Not applicable.

\section{Competing interests}

The authors declare that they have no competing interests.

\section{Author details}

${ }^{1}$ International Nanobody Research Center of Guangxi, College of Stomatology, Guangxi Medical University, Nanning, Guangxi 530021, China. ${ }^{2}$ Laboratory of Tropical Biomedicine and Biotechnology, School of Tropical Medicine and Laboratory Medicine, Hainan Medical University, Haikou 570100, Hainan, China. ${ }^{3}$ Department of traditional Chinese medicine, The First Affiliated Hospital of Hainan Medical College, Haikou 570100, Hainan, China. ${ }^{4}$ Department of Anesthesiology, Tunchang people's Hospital, Tunchang 571600, Hainan, China. ${ }^{5}$ Department of Anatomy, Zunyi Medical University, Zunyi 563006, China. ${ }^{6}$ Department of acupuncture and moxibustion, Hainan General Hospital, The Affiliated Hainan Hospital of Hainan Medical University, Haikou 570100, Hainan, China. ${ }^{7}$ Department of Laboratory Medicine, The second affiliated hospital of Hainan medical university, Haikou 570311, Hainan, China. ${ }^{8}$ Affiliated Tumor Hospital, Guangxi Medical University, Nanning 530021, Guangxi, China.

Received: 21 January 2021 Accepted: 26 August 2021

Published online: 15 September 2021

\section{References}

1. Rosenberg SA. Progress in human tumour immunology and immunotherapy. Nature. 2001;411(6835):380-4. https://doi.org/10.1038/3 5077246.

2. Schmidt-Wolf IGH, Negrin RS, Kiem HP. Use of a SCID mouse/human lymphoma model to evaluate cytokine-induced killer with potent antitumor cell activity. J Exp Med. 1991;174(1):139-49. https://doi.org/10.1084/jem.1 74.1.139.

3. Wang $Y, X u Z$, Zhou F, Sun Y, Chen J, Li L. The combination of dendritic cells-cytotoxic $T$ lymphocytes/cytokine-induced killer (DC-CTL/CIK) therapy exerts immune and clinical responses in patients with malignant tumors. Exper Hematol Oncol. 2014;4(1):32.

4. Schmeel LC, Schmeel FC, Coch C, Schmidt-Wolf IGH. Cytokine-induced killer (CIK) cells in cancer immunotherapy: report of the international registry on CIK cells (IRCC). J Cancer Res Clin Oncol. 2015;141(5):839-49. https://doi. org/10.1007/s00432-014-1864-3.

5. Wu T, Dai Y. Tumor microenvironment and therapeutic response. Cancer Lett. 2016:387:61-8.

6. Ruella M, Klichinsky M, Kenderian SS, Shestova O, Ziober A, Kraft DO, et al. Overcoming the immunosuppressive tumor microenvironment of Hodgkin lymphoma using chimeric antigen receptor T cells. Cancer Discovery. 2017; 7(17):1154-67. https://doi.org/10.1158/2159-8290.CD-16-0850.

7. Bardoli AD, Afshar M, Viney R, et al. The PD-1/PD-L1 axis in the pathogenesis of urothelial bladder cancer and evaluating its potential as a therapeutic target. Future Oncol. 2016;12(5):595-600. https://doi.org/10.2217/ fon.15.337. 
8. Brunet JF, Denizot F, Luciani MF, Roux-Dosseto M, Suzan M, Mattei MG, et al. A new member of the immunoglobulin superfamily-CTLA-4. Nature. 1987;328(6127):267-70. https://doi.org/10.1038/328267a0.

9. Walunas TL, Lenschow DJ, Bakker CY, Linsley PS, Freeman GJ, Green JM, et al. CTLA-4 can function as a negative regulator of T cell activation. Immunity. 1994;1(5):405-13. https://doi.org/10.1016/1074-7613(94)90071-X

10. Tivol EA, Borriello F, Schweitzer AN, Lynch WP, Bluestone JA, Sharpe AH. Loss of CTLA-4 leads to massive lymphoproliferation and fatal multiorgan tissue destruction, revealing a critical negative regulatory role of CTLA-4. Immunity. 1995;3(5):541-7. https://doi.org/10.1016/1074-7613(95)90125-6.

11. Callahan MK, Postow MA, Wolchok JD. CTLA-4 and PD-1 pathway blockade: combinations in the clinic. Front Oncol. 2014;4:385. https://doi.org/10.3389/ fonc.2014.00385.

12. Wu X, Giobbie-Hurder A, Connolly EM, et al. Anti-CTLA-4 based therapy elicits humoral immunity to galectin-3 in patients with metastatic melanoma. Oncoimmunology. 2018;7(7):01-25.

13. Rosskopf S, Leitner J, Zlabinger GJ, Steinberger P. CTLA-4 antibody ipilimumab negatively affects CD4 ${ }^{+} \mathrm{T}$-cell responses in vitro. Cancer Immunol Immunother: Cll. 2019;68(8):1359-68. https://doi.org/10.1007/s002 62-019-02369-x.

14. Hamers-Casterman C, Atarhouch T, Muyldermans S, Robinson G, Hamers C, Songa EB, et al. Naturally occurring antibodies devoid of light chains. Nature. 1993;363(6428):446-8. https://doi.org/10.1038/363446a0.

15. Muyldermans S. Nanobodies: natural single-domain antibodies. Annu Rev Biochem. 2013;82(82):775-97. https://doi.org/10.1146/annurev-biochem-063 011-092449.

16. De Genst E, Silence K, Decanniere K, Conrath K, Loris R, Kinne J, et al. Molecular basis for the preferential cleft recognition by dromedary heavychain antibodies. Proc Natl Acad Sci U S A. 2006;103(12):4586-91. https:// doi.org/10.1073/pnas.0505379103.

17. Wan R, Liu A, Hou X, Lai Z, Li J, Yang N, et al. Screening and antitumor effect of an anti-CTLA-4 nanobody. Oncol Rep. 2018;39(2):511-8. https://doi. org/10.3892/or.2017.6131.

18. Wu W, Hou X, Yang $X$, et al. Highly sensitive detection of CTLA-4-positive Tcell subgroups based on nanobody and fluorescent carbon quantum dots. Oncol Lett. 2019;18:109-16.

19. Zhang W, Song Z, Xiao J, Liu X, Luo Y, Yang Z, et al. Blocking the PD-1/PDL1 axis in dendritic cell-stimulated cytokine-induced killer cells with pembrolizumab enhances their therapeutic effects against hepatocellular carcinoma. J Cancer. 2019;10(11):2578-87. https://doi.org/10.7150/jca.26961.

20. Lissoni P, Brivio F, Fumagalli L, Messina G, Meregalli S, Porro G, et al. Effects of the conventional antitumor therapies surgery, chemotherapy, radiotherapy and immunotherapy on regulatory $T$ lymphocytes in cancer patients. Anticancer Res. 2009:29(5):1847-52.

21. Singh C, Qian JM, Yu JB, Chiang VL. Local tumor response and survival outcomes after combined stereotactic radiosurgery and immunotherapy in non-small cell lung cancer with brain metastases. J Neurosurg. 2019;132(2): 512-7. https://doi.org/10.3171/2018.10.JNS181371.

22. Niam M, Linn YC, Chong SF, et al. Clinical scale expansion of cytokineinduced killer cells is feasible from healthy donors and patients with acute and chronic myeloid leukemia at various stages of therapy. Exp Hematol. 2011;39(9):897-903. https://doi.org/10.1016/j.exphem.2011.06.005.

23. Zhaohu Y. Huikuan, et al. combined induction with anti-PD-1 and antiCTLA-4 antibodies provides synergistic antitumor effects in DC-CIK cells in renal carcinoma cell lines. Int J Clin Exp Pathol. 2019;12(1):123-32.

24. Rui T, Cheng X, Wu H, Wang F, Ye Z, Wu G. Lentiviral delivery of CTLA4 shRNA improves the expansion of cytokineinduced killer cells and enhances cytotoxic activity in vitro. Oncol Lett. 2018;15(1):741-6. https://doi.org/10.3 892/ol.2017.7376.

25. Oyen D, Srinivasan V, Steyaert J, Barlow JN. Constraining enzyme conformational change by an antibody leads to hyperbolic inhibition. J Mol Biol. 2011;407(1):138-48. https://doi.org/10.1016/j.jmb.2011.01.017.

26. Bever CS, Dong JX, Vasylieva N, Barnych B, Cui Y, Xu ZL, et al. VHH antibodies: emerging reagents for the analysis of environmental chemicals. Anal Bioanal Chem. 2016;408(22):5985-6002. https://doi.org/10.1007/s00216016-9585-x.

27. Zhang Q, Liu XY, Zhang T, Zhang XF, Zhao L, Long F, et al. The dualfunctional capability of cytokine-induced killer cells and application in tumor immunology. Hum Immunol. 2015;76(5):385-91. https://doi.org/10.1 016/j.humimm.2014.09.021.
28. Yufeng D, Guocheng Z, Dongliang X, Rong F, Yuhong C, Ruying $L$, et al. Whole-tumor-antigen-pulsed dendritic cells elicit cytotoxic T-cell response against pediatric nasopharyngeal carcinoma in vitro. Med Oncol. 2009;26(1): 78-85. https://doi.org/10.1007/s12032-008-9093-8.

\section{Publisher's Note}

Springer Nature remains neutral with regard to jurisdictional claims in published maps and institutional affiliations.
Ready to submit your research? Choose BMC and benefit from:

- fast, convenient online submission

- thorough peer review by experienced researchers in your field

- rapid publication on acceptance

- support for research data, including large and complex data types

- gold Open Access which fosters wider collaboration and increased citations

- maximum visibility for your research: over $100 \mathrm{M}$ website views per year

At $\mathrm{BMC}$, research is always in progress.

Learn more biomedcentral.com/submissions 